\title{
Signal transducer and activator of transcription 3 regulates CCAAT-enhancer-binding homologous protein expression in osteoblasts through upregulation of microRNA-205
}

\author{
JIAN ZHUANG, RUFENG GAO, HAIHUI WU, XIAO WU and FUGEN PAN \\ Department of Orthopedics, Qingpu Branch of Zhongshan Hospital, Fudan University, Shanghai 201700, P.R. China
}

Received June 5, 2014; Accepted December 18, 2014

DOI: $10.3892 /$ etm.2015.2464

\begin{abstract}
The transcription factor, CCAAT-enhancer-binding protein homologous protein (CHOP), is induced by endoplasmic reticulum-stress and mediates programmed cell death. In osteoblasts, CHOP overexpression increases the rate of apoptosis, leading to osteoblastic dysfunction. However, the regulatory mechanisms underlying CHOP expression remain unclear. In the present study, western blot analysis was used to demonstrate that the activation of signal transducer and activator of transcription 3 (STAT3) inhibited the levels of the CHOP protein, whereas small interfering RNA-mediated the knockdown of STAT3 upregulated CHOP expression. Furthermore, STAT3 was shown to increase the expression level of microRNA (miR)-205. A luciferase reporter assay revealed that miR-205 was able to directly target the 3'-untranslated region of the CHOP gene to inhibit its protein expression. The miR-205 antisense largely abolished the inhibitory effect of STAT3 activation on the levels of CHOP protein. Therefore, the results demonstrated a previously unknown STAT3/miR-205/CHOP signaling pathway in osteoblasts, which may aid the understanding of the pathogenic mechanisms of associated diseases, including osteoporosis.
\end{abstract}

\section{Introduction}

Osteoblasts are derived from undifferentiated mesenchymal stem cells and play a key role in bone formation (1). Therefore, investigation into the expression levels and functions of master regulators is essential in order to reveal the mechanisms underlying osteoblast differentiation.

Correspondence to: Dr Fugen Pan, Department of Orthopedics, Qingpu Branch of Zhongshan Hospital, Fudan University, 1158 Park East Road, Shanghai 201700, P.R. China

E-mail: panfgen@126.com

Key words: CCAAT-enhancer-binding homologous protein, signal transducer and transcription activator 3, microRNA-205, osteoporosis
CCAAT-enhancer-binding homologous protein (CHOP), also known as growth arrest and DNA damage-inducible gene 153 and DNA damage inducible transcript 3, is a member of the basic leucine zipper family of transcription factors (2). CHOP regulates mRNA transcription indirectly by interacting with various nuclear proteins, including CCAAT/enhancer-binding protein- $\beta$, activating transcription factor, cAMP response element-binding protein and Fos/Jun (3-5). CHOP is implicated in apoptosis, which may be induced by cellular stress, including endoplasmic reticulum (ER) stress $(6,7)$. Transgenic mice overexpressing CHOP have shown reduced trabecular bone volume due to decreased bone formation, resulting in osteopenia (8). Furthermore, a previous study demonstrated that hyperglycemia induced ER stress-dependent CHOP expression in osteoblasts (9). CHOP expression in osteoblasts obtained from diabetic rats or cultured in a high-glucose medium is elevated (9), suggesting that abnormal expression of CHOP may play an important role in the pathogenesis of osteoporosis. However, the regulatory mechanisms underlying $\mathrm{CHOP}$ expression remain largely unknown.

Signal transducer and activator of transcription 3 (STAT3) is a transcription factor that promotes cell survival and differentiation $(10,11)$. The aim of the present study was to investigate the effects of STAT3 activation on the expression of CHOP protein in osteoblasts. In addition, the effect of miRNA (miR)-205 was analyzed in order to determine its role in the STAT3-mediated regulation of $\mathrm{CHOP}$ protein expression. Potential miRNAs that were able to regulate CHOP expression were analyzed by bioinformatic software (data not shown) and (miR)-205 was selected for further analysis.

\section{Materials and methods}

Cell culture. MC3T3-E1 cells were obtained from the Cell Bank of the Chinese Academy of Sciences (Shanghai, China), and were maintained in a modified Eagle's minimal essential medium (Invitrogen Life Technologies, Carlsbad, CA, USA) containing $100 \mathrm{U} / \mathrm{ml}$ penicillin and $100 \mu \mathrm{g} / \mathrm{ml}$ streptomycin (Invitrogen Life Technologies). Mouse bone marrow mesenchymal stem cells (MSCs) were prepared from the bone marrow of femurs and tibias harvested from seven-week-old male C57B/L6 mice (12). Interleukin (IL)-6 was purchased 
from Merck Sharpe \& Dohme (Shanghai, China). The present study was approved by the Ethics Committee of the Qingpu Branch of Zhongshan Hospital, Fudan University (Shanghai, China).

Small interfering RNA (siRNA) oligos and miRNA. siRNA oligos targeting green fluorescent protein (GFP) or STAT3 were designed and synthesized by GenePharm, Inc. (Shanghai, China). siRNA targeting GFP was used as a negative control for STAT3 siRNA. The negative control for miR-205 mimics and antisense was purchased from Guangzhou RiboBio Co., Ltd. (Guangdong, China).

Plasmids, transient transfections and luciferase assays. An miR-205 promoter (420 bp) was amplified from the mouse genomic DNA template and inserted into a pGL3 vector (Promega Corporation, Madison, WI, USA). A mutant STAT3 binding site was generated using a polymerase chain reaction (PCR) mutagenesis kit (Toyobo Co., Ltd., Osaka, Japan) with the primer, 5'-GATTCAGGGACATAAAACCAATAC-3' (mutation site, AAAA), and a reverse complementary primer. Reporter vectors carrying the miR-205 target site were constructed by synthesizing a 3'-untranslated region (UTR) fragment containing the predicted target sites (miRWalk) (13) for $\mathrm{CHOP}$ cDNA, and subsequently inserting the $\mathrm{CHOP}$ cDNA fragment into the multiple cloning site of a pMIR-REPORT ${ }^{\mathrm{TM}}$ luciferase miRNA expression reporter vector (Ambion Life Technologies, Carlsbad, CA, USA). Transient transfection was performed using Lipofectamine ${ }^{\circledR} 2000$ (Invitrogen Life Technologies), according to the manufacturer's instructions. For the luciferase reporter assay, MC3T3-E1 cells were seeded in 24-well plates and transfected with the indicated plasmids. Cells were harvested $36 \mathrm{~h}$ after transfection. Luciferase activity was measured using the Dual-Luciferase ${ }^{\circledR}$ Reporter Assay System (Promega Corporation).

RNA extraction, quantitative analysis and western blot analysis. Total RNA was isolated from the tissues or cells with TRIzol reagent (Invitrogen Life Technologies), and reverse transcription was performed using a Takara RNA PCR kit (Takara Biotechnology Co., Ltd., Dalian, China), following the manufacturer's instructions. In order to quantify the transcripts of the genes of interest, quantitative PCR was performed using SYBR Green Premix Ex Taq (Takara Bio, Inc., Otsu, Japan) and a Light Cycler 480 (Roche Diagnostics, Basel, Switzerland). The primer sequences for the CHOP gene were as follows: forward 5'- AAGCCTGGTATGAGGATCTGC-3' and reverse: 5'-TTCCTGGGGATGAGATAT AGGTG-3'. The PCR conditions included an initial holding period at $94^{\circ} \mathrm{C}$ for $5 \mathrm{~min}$, followed by a two-step PCR program of $94^{\circ} \mathrm{C}$ for $10 \mathrm{sec}$ and $60^{\circ} \mathrm{C}$ for $45 \mathrm{sec}$ for 45 cycles. For western blot analysis, tissues and cells were lysed in radioimmunoprecipitation buffer containing $50 \mathrm{mM}$ Tris- $\mathrm{HCl}, 150 \mathrm{mM}$ $\mathrm{NaCl}, 5 \mathrm{mM} \mathrm{MgCl}{ }_{2}, 2 \mathrm{mM}$ EDTA, $1 \mathrm{mM} \mathrm{NaF}, 1 \% \mathrm{NP} 40$ and $0.1 \%$ SDS. The antibodies used were monoclonal rabbit anti-STAT3 (D3Z2G; 1:1,000; \#12640; Cell Signaling Technology, Inc., Danvers, MA, USA), monoclonal mouse anti-CHOP (1:2,000; \#ab11419; Abcam, Cambridge, UK) and monoclonal mouse anti-GAPDH (1:5,000; \#sc-365062; Santa Cruz Biotechnology, Inc., Dallas, TX, USA).
Chromatin immunoprecipitation (ChIP) assays. ChIP assay kits were purchased from Upstate Biotechnology, Inc. (New York, NY, USA). MC3T3-E1 cells were fixed with formaldehyde. DNA was sheared into 200-1,000-bp fragments using sonication. Chromatin was incubated and precipitated with the STAT3 antibody or IgG (Santa Cruz Biotechnology, Inc.).

Statistical analysis. Values are expressed as the mean \pm standard error of the mean. Statistical analyses were performed with Graphpad software, version 5.0 (La Jolla, CA, USA). The two-tailed Student's t-test was used to evaluate the statistical significance of the differences between the two groups. Values of ${ }^{*} \mathrm{P}<0.05,{ }^{* *} \mathrm{P}<0.01$ or ${ }^{* * *} \mathrm{P}<0.001$ were considered to indicate a statistically significant difference.

\section{Results}

IL- 6 treatment and STAT3 overexpression downregulate CHOP protein levels. To assess whether STAT3 regulated CHOP expression, MC3T3-E1 cells were treated with IL-6, a known STAT3 agonist. As shown in Fig. 1A, IL-6 treatment did not affect CHOP mRNA expression. However, the protein expression levels were notably reduced, accompanied by enhanced phosphorylation of STAT3 (Fig. 1B). Similar results were observed in the primary mouse bone marrow MSCs (Fig. 1C and D). To rule out the potential non-specific effects of IL-6, these two cell types were transfected with lentiviruses containing an empty vector or constitutively-activated (CA)-STAT3 (14). The results indicated that CA-STAT3 also inhibited protein expression of CHOP, while the mRNA expression levels remained unaffected (Fig. 2).

STAT3 inhibition by siRNA oligos increases CHOP expression. Endogenous STAT3 expression was inhibited by siRNA oligos. Knockdown of STAT3 increased CHOP protein levels in the MC3T3-E1 and primary mouse bone marrow MSCs (Fig. 3). These results demonstrated that STAT3 may be a negative regulator in the control of $\mathrm{CHOP}$ expression in osteoblasts.

Regulation of miR-205 by STAT3 activation. STAT3 activation was found to regulate CHOP protein expression, but not mRNA expression. Thus, it was hypothesized that STAT3 regulates CHOP expression at a translational level. miRNAs are known to recognize and bind to the target 3'-UTR of mRNAs, which leads to mRNA degradation or translational inhibition of the target mRNA and downregulation of target gene expression $(15,16)$. Using the miRWalk algorithm based on seed recognition, a number of miRNAs were identified that potentially interacted with the CHOP transcript (data not shown). However, only miR-205 was notably elevated in the MC3T3-E1 cells treated with IL-6 or transfected with CA-STAT3 (Fig. 4A and B). Targeted knockdown of endogenous STAT3 also reduced miR-205 expression (Fig. 4C), suggesting that STAT3 activation increases the levels of miR-205.

In order to investigate whether STAT3 is a transcriptional activator of miR-205, MC3T3-E1 cells were transfected with a reporter vector encoding luciferase, under the control of the miR-205 promoter. Concurrent expression of CA-STAT3 with 

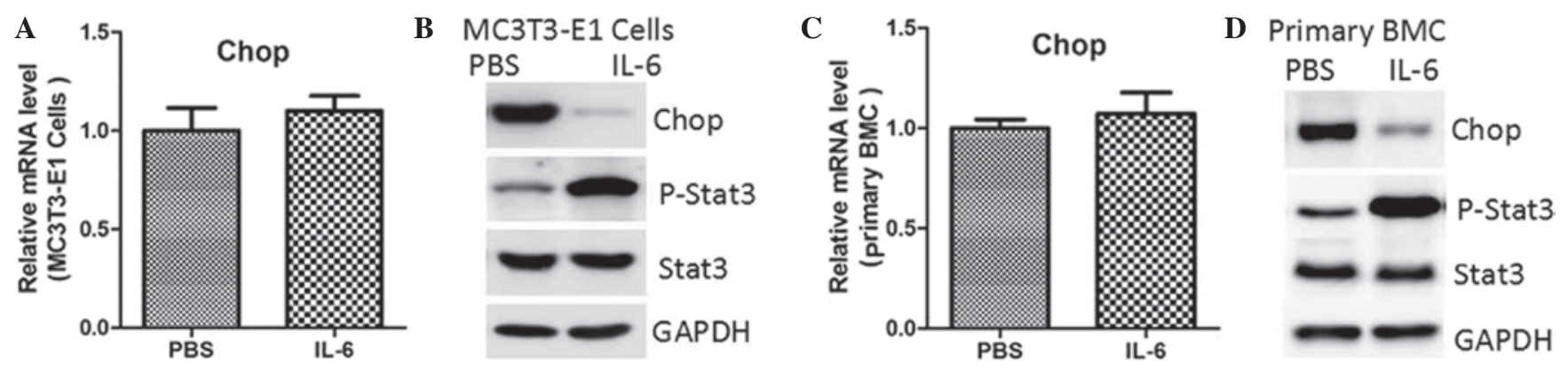

Figure 1. Downregulation of CHOP protein levels by IL-6. The mRNA and protein expression levels of CHOP were determined by quantitative polymerase chain reaction and western blot analysis, respectively, in (A and B) MC3T3-E1 cells and (C and D) primary mouse BMCs treated with PBS or IL-6 (10 ng/ml) for 24 and 48 h. CHOP, CCAAT-enhancer-binding homologous protein; STAT3, signal transducer and activator of transcription 3; P-STAT3, phosphorylated-STAT3; PBS, phosphate-buffered saline; IL-6, interleukin-6; BMC, bone marrow cell.
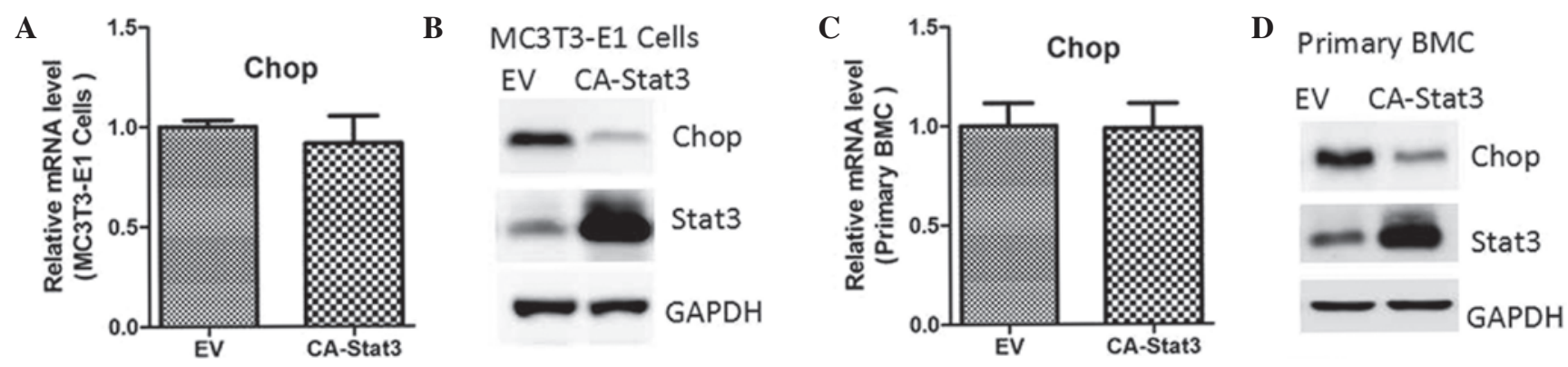

Figure 2. STAT3 overexpression reduces CHOP protein content in osteoblasts. The mRNA and protein expression levels of CHOP were determined by quantitative polymerase chain reaction and western blot analysis, respectively, in (A and B) MC3T3-E1 cells and (C and D) primary mouse BMCs transfected with lentiviruses containing an EV or CA-STAT3 for 24 and 48 h. CHOP, CCAAT-enhancer-binding homologous protein; EV, empty vector; CA-STAT3, constitutively active-signal transducer and activator of transcription 3; BMC, bone marrow cell.
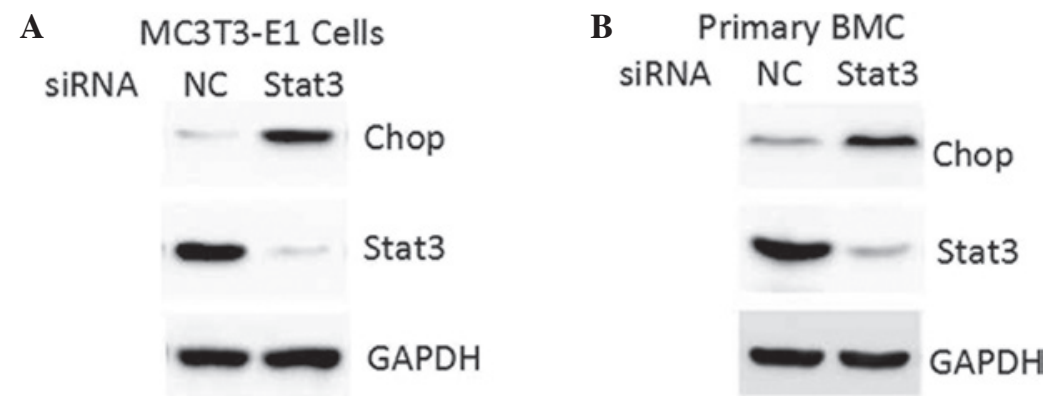

Figure 3. Protein expression levels of CHOP and STAT3 were determined by quantitative polymerase chain reaction and western blot analysis in (A) MC3T3-E1 cells and (B) mouse primary BMCs transfected with siRNA oligos targeting STAT3 or NC. CHOP, CCAAT-enhancer-binding homologous protein; STAT3, signal transducer and activator of transcription 3; NC, negative control; BMC, bone marrow cell.

the miR-205 reporter construct enhanced miR-205 promoter activity (Fig. 4D), which was inhibited by mutation of the STAT3 DNA-binding site in the miR-205 promoter (Fig. 4D). In addition, ChIP assays were performed to assess whether STAT3 directly binds to the miR-205 promoter. Levels of STAT3 protein bound to the miR-205 promoter were significantly increased in the MC3T3-E1 cells treated with IL-6 (Fig. 4E). Therefore, the results indicated that miR-205 may be a transcriptional target of STAT3 in osteoblasts.

miR-205 directly targets the 3'-UTR of the CHOP gene in osteoblasts. To confirm CHOP as the target gene of miR-205, a fragment of the CHOP gene containing the binding sites for miR-205 was engineered into a luciferase reporter vector and luciferase reporter activity assays were conducted.
Transfection of miR-205 caused a substantial reduction in luciferase activity in the luciferase expression constructs carrying the target fragment (Fig. 5A). Furthermore, the repressive effect of miR-205 on the CHOP 3'-UTR was inhibited by point mutations in the miR-205-binding site region of the CHOP 3'-UTR (Fig. 5A).

Ectopic expression of miR-205 decreased the protein expression levels of $\mathrm{CHOP}$ in MC3T3-E1 and mouse primary bone marrow cells (Fig. $5 \mathrm{~B}$ and $\mathrm{C}$ ), while the inhibition of miR-205 was observed to upregulate CHOP protein levels (Fig. 5D and E). Furthermore, miR-205 antisense negated the inhibitory effect of STAT3 activation on CHOP protein levels (Fig. 5F). Therefore, the results suggested that STAT3 negatively regulated $\mathrm{CHOP}$ protein expression through the upregulation of miR-205 in osteoblasts. 

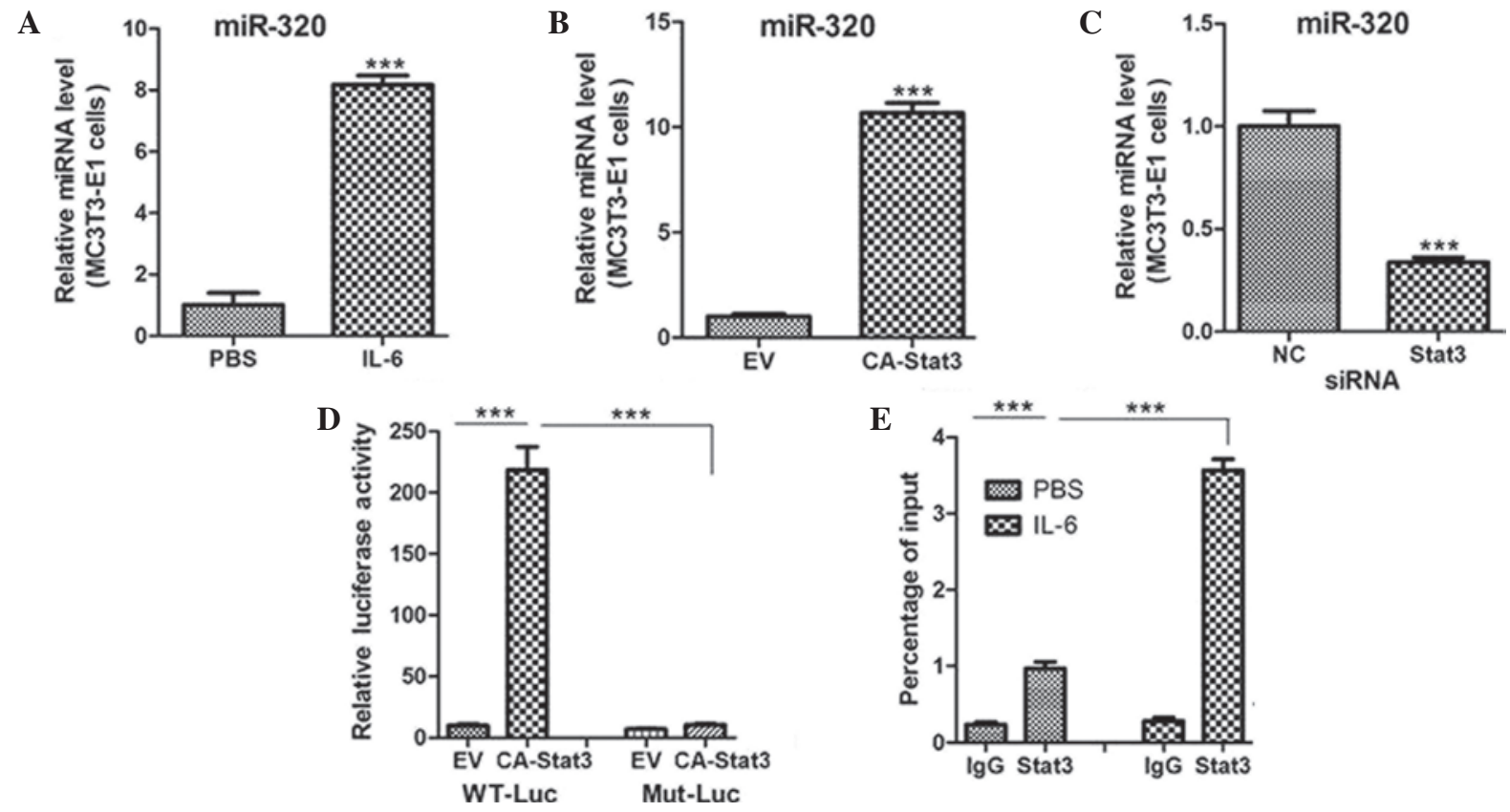

Figure 4. STAT3 activation transcriptionally regulates miR-205 expression. Quantitative polymerase chain reaction (PCR) analysis of miR-205 expression in MC3T3-E1 cells treated with (A) PBS or IL-6 (10 ng/ml) for $12 \mathrm{~h}$, (B) transfected with an EV or CA-STAT3 and (C) transfected with siRNA oligos targeting STAT3 or NC. (D) Relative luciferase activity of MC3T3-E1 cells cotransfected with WT or Mut miR-205 promoters and EV or CA-STAT3. (E) Quantitative PCR analysis of chromatin immunoprecipitation assays showing STAT3 binding to the miR-205 promoter. Chromatins were prepared from miR-205 cells treated with PBS or IL-6 (10 ng/ml). ${ }^{* * * *} \mathrm{P}<0.001$. miR, microRNA; siRNA, small interfering RNA; PBS, phosphate-buffered saline; IL-6, interleukin-6; EV, empty vector; CA-STAT3, constitutively active-signal transducer and activator of transcription 3; NC, negative control; WT, wild type; Mut, mutant.

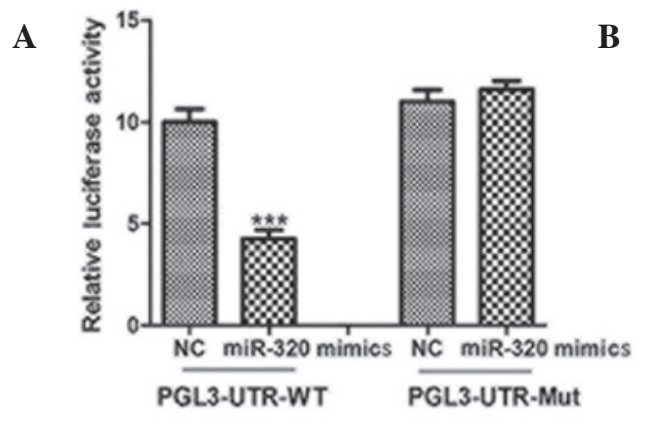

D

$$
\begin{aligned}
& \text { Primary BMC } \\
& \text { NC miR-320-antisense }
\end{aligned}
$$

Chop

\section{GAPDH}

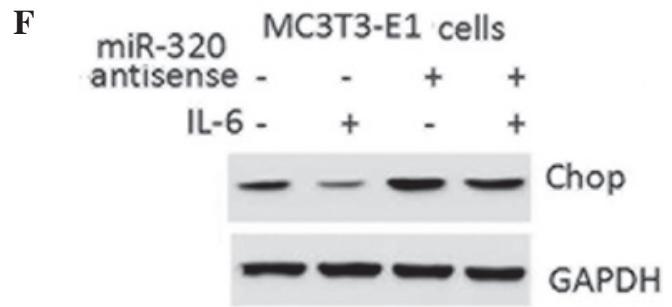

MC3T3-E1 cells

NC miR-320 mimics

- Chop

GAPDH

C Primary BMC

E

MC3T3-E1 cells

NC miR-320-antisense

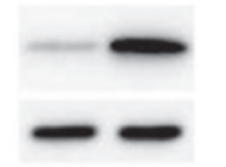

Chop

\section{GAPDH}

G

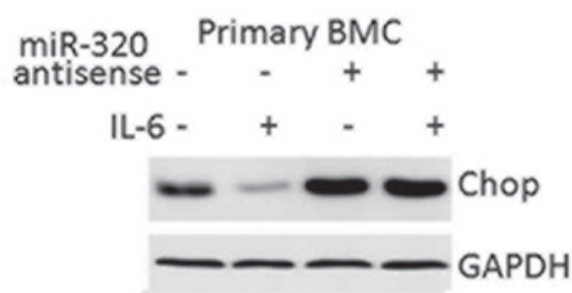

Figure 5. miR-205 negatively regulates CHOP protein expression through targeting its 3'-UTR. (A) Relative luciferase activity of WT or Mut CHOP 3'-UTR in MC3T3-E1 cells transfected with NC or miR-205 mimics. Western blot analysis of CHOP protein expression in (B) MC3T3-E1 cells and (C) mouse primary BMCs transfected with NC or miR-205 mimics, (D) MC3T3-E1 and (E) mouse primary BMCs transfected with NC or miR-205 antisense and in (F) MC3T3-E1 and $(\mathrm{G})$ mouse primary BMCs pretransfected with NC or miR-205 antisense for $24 \mathrm{~h}$ and then treated with PBS or IL-6 (10 ng/ml) for $24 \mathrm{~h}$. ${ }^{* * *} \mathrm{P}<0.001, \mathrm{vs}$. NC. NC, negative control; miR, microRNA; UTR, untranslated region; CHOP, CCAAT-enhancer binding homologous protein; IL-6, interleukin-6; WT, wild type; Mut, mutant; PBS, phosphate-buffered saline; BMC, bone marrow cell. 


\section{Discussion}

In the present study, STAT3 was shown to repress $\mathrm{CHOP}$ protein expression in osteoblasts. At a molecular level, miR-205 was found to be a transcriptional target of STAT3. miR-205 directly targeted the CHOP 3'-UTR, suggesting that CHOP is regulated by miR-205 in osteoblasts. Collectively, the present observations indicate that activation of STAT3 may inhibit CHOP expression through upregulation of miR-205. STAT3 activation has been reported to promote osteoblast formation and protect against ethanol-induced bone loss $(17,18)$. Therefore, the present study proposes a novel mechanism for the protective roles of STAT3 in osteoblasts.

A previous study showed that miR-205 negatively regulates androgen receptors and is inversely correlated to the occurrence of metastases and shortened overall survival in prostate cancer patients (19). However, miR-205 expression is significantly upregulated in human endometrial endometrioid carcinoma tissues, which promotes tumor proliferation and invasion by targeting estrogen-related receptors (20). Therefore, miR-205 may function as a tumor suppressor or as an oncogene, depending on the cellular context. Furthermore, miR-205 is expressed in a lineage-related pattern in mesenchymal cell types and is regulated by runt-related transcription factor-2, a master regulator of osteoblast differentiation (21). Therefore, the function of miR-205 in osteoblasts and bone formation should be investigated further in future studies.

Timofeeva et al (22) demonstrated that STAT3 suppresses mRNA transcription of the CHOP gene (22). The authors demonstrated that the STAT3-binding region of the $\mathrm{CHOP}$ promoter was localized in the DNase I hypersensitive site of chromatin in cancer cells, but not in non-transformed cells, indicating that STAT3 binding and suppressive action may be chromatin structure-dependent (22). In accordance with these observations, the regulation of CHOP by STAT3 has been hypothesized to be cell- or tissue-specific.

In summary, the current study demonstrated that STAT3 activation reduces $\mathrm{CHOP}$ protein levels in osteoblasts, which is partly dependent on the upregulation of miR-205. Further investigation into this osteoblast signaling pathway may aid the understanding of the pathogenic mechanisms underlying osteoblast-related diseases, including osteoporosis.

\section{Acknowledgements}

This study was supported by grants from the Shanghai Key Medical Specialty Construction, Orthopedic Trauma (no. ZK 2012A36) and the Leading Discipline Program of the Qingpu Branch of Zhongshan Hospital, Fudan University, (no. WL 2011-01).

\section{References}

1. DiGirolamo DJ, Clemens TL and Kousteni S: The skeleton as an endocrine organ. Nat Rev Rheumatol 8: 674-683, 2012.

2. Ramji DP and Foka P: CCAAT/enhancer-binding proteins: structure, function and regulation. Biochem J 365: 561-575, 2002.

3. Tabas I and Ron D: Integrating the mechanisms of apoptosis induced by endoplasmic reticulum stress. Nat Cell Biol 13: 184-190, 2011.

4. Malhi $\mathrm{H}$ and Kaufman RJ: Endoplasmic reticulum stress in liver disease. J Hepatol 54: 795-809, 2011.

5. Scull CM and Tabas I: Mechanisms of ER stress-induced apoptosis in atherosclerosis. Arterioscler Thromb Vasc Biol 31: 2792-2797, 2011.

6. Schönthal AH: Pharmacological targeting of endoplasmic reticulum stress signaling in cancer. Biochem Pharmacol 85: 653-666, 2013

7. Eizirik DL, Miani M and Cardozo AK: Signalling danger: endoplasmic reticulum stress and the unfolded protein response in pancreatic islet inflammation. Diabetologia 56: 234-241, 2013.

8. Pereira RC, Stadmeyer LE, Smith DL, Rydziel S and Canalis E: CCAAT/Enhancer-binding protein homologous protein (CHOP) decreases bone formation and causes osteopenia. Bone 40: 619-626, 2007.

9. Liu W, Zhu X, Wang Q and Wang L: Hyperglycemia induces endoplasmic reticulum stress-dependent CHOP expression in osteoblasts. Exp Ther Med 5: 1289-1292, 2013.

10. Taub R: Liver regeneration: from myth to mechanism. Nat Rev Mol Cell Biol 5: 836-847, 2004.

11. Yu H, Pardol D and Jove R: STATs in cancer inflammation and immunity: a leading role for STAT3. Nat Rev Cancer 9: 798-809, 2009.

12. Edgar CM, Chakravarthy V, Barnes G, et al: Autogenous regulation of a network of bone morphogenetic proteins (BMPs) mediates the osteogenic differentiation in murine marrow stromal cells. Bone 40: 1389-1398, 2007.

13. miRWalk: The database on predicted and validated microRNA targets. http://www.umm.uni-heidelberg.de/apps/zmf/mirwalk/. Accessed March 20, 2012

14. Lee H, Herrmann A, Deng JH, et al: Persistently activated Stat3 maintains constitutive NF-kappaB activity in tumors. Cancer Cell 15: 283-293, 2009.

15. Ameres SL and Zamore PD: Diversifying microRNA sequence and function. Nat Rev Mol Cell Biol 14: 475-488, 2013.

16. Sun K and Lai EC: Adult-specific functions of animal microRNAs. Nat Rev Genet 14: 535-548, 2013.

17. Nicolaidou V, Wong MM, Redpath AN, et al: Monocytes induce STAT3 activation in human mesenchymal stem cells to promote osteoblast formation. PLoS One 7: e39871, 2012.

18. Chen JR, Shankar K, Nagarajan S, Badger TM and Ronis MJ: Protective effects of estradiol on ethanol-induced bone loss involve inhibition of reactive oxygen species generation in osteoblasts and downstream activation of the extracellular signal-regulated kinase/signal transducer and activator of transcription 3/receptor activator of nuclear factor-kappaB ligand signaling cascade. J Pharmacol Exp Ther 324: 50-59, 2008.

19. Hagman Z, Haflidadóttir BS, et al: miR-205 negatively regulates the androgen receptor and is associated with adverse outcome of prostate cancer patients. Br J Cancer 108: 1668-1676, 2013.

20. Su N, Qiu H, Chen Y, Yang T, Yan Q and Wan X: miR-205 promotes tumor proliferation and invasion through targeting ESRRG in endometrial carcinoma. Oncol Rep 29: 2297-2302, 2013.

21. Zhang Y, Xie RL, Croce CM, et al: A program of microRNAs controls osteogenic lineage progression by targeting transcription factor Runx2. Proc Natl Acad Sci USA 108: 9863-9868, 2011.

22. Timofeeva OA, Tarasova NI, Zhang X, et al: STAT3 suppresses transcription of proapoptotic genes in cancer cells with the involvement of its N-terminal domain. Proc Natl Acad Sci USA 110: 1267-1272, 2013. 